\title{
RIESGOS PSICOSOCIALES EN EL TRABAJO Y SALUD OCUPACIONAL
}

\begin{abstract}
Pedro R. Gil-Monte ${ }^{1, a}$
RESUMEN

Los cambios que han ocurrido en las últimas décadas sobre los procesos laborales y el diseño del trabajo son de carácter sociodemográfico, económico, político, y tecnológico. Estos cambios han originado nuevos riesgos psicosociales en el trabajo que afectan a la salud y la calidad de vida laboral, pues incrementan los niveles de estrés de los trabajadores. El objetivo del estudio es presentar este tipo de riesgos, sus consecuencias, y algunas recomendaciones para promover la salud en el trabajo como estrategia para mejorar la salud pública de la población. El estudio se estructura en cinco puntos en los que: (1) se presenta el concepto de factores y riesgos psicosociales en el trabajo, (2) se describen los principales riesgos psicosociales laborales emergentes, (3) se ofrecen algunos datos sobre la prevalencia de los riesgos psicosociales en el trabajo en Europa y de sus consecuencias, (4) se presentan algunas recomendaciones sobre promoción de la salud en el lugar de trabajo, y (5) se describe el objetivo la Psicología de la Salud Ocupacional y se concluye con la recomendación de fomentar la salud psicosocial en el lugar de trabajo como estrategia para mejorar la salud pública de la población.
\end{abstract}

Palabras clave: Salud ocupacional; Psicología; Estrés laboral; Enfermedades ocupacionales (fuente: DeCS BIREME).

\section{PSYCHOSOCIAL RISKS AT WORK AND OCCUPATIONAL HEALTH}

\begin{abstract}
The changes on work processes and job design in recent decades are focused in the demographic, economic, political, and technological aspects. These changes have created new psychosocial risks at work that affect the health and quality of workplace, increasing stress levels among workers. The aim of this study is to present such risks, their consequences, and some recommendations to promote health at the workplace as a strategy to improve public health of the population. The study is divided into five points in which: (1) introduces the concept of risk factors and psychosocial work, (2) describes the main emerging psychosocial risks labor, (3) provides some information on the prevalence of psychosocial risks at work in Europe and its consequences, (4) recommendations for health promotion in the workplace, and (5) describes the objective of Occupational Health Psychology and concludes with the recommendations to promote psychosocial health in the workplace as a strategy to improve public health of the population.
\end{abstract}

Key words: Occupational health; Psychology; Burnout, professional; Occupational diseases (source: MeSH NLM).

\section{RIESGOS PSICOSOCIALES EN EL TRABAJO Y SALUD OCUPACIONAL}

El mundo laboral es un fenómeno complejo y cambiante. En el informe del año 2006 de la Conferencia Internacional del Trabajo se identifican cuatro fuerzas como los principales motores del cambio en el mundo del trabajo: 1) el imperativo del desarrollo; 2) la transformación tecnológica; 3) la intensificación de la competencia a escala mundial; y 4) la mayor orientación en función de los mercados, y una función más reducida del Estado. Junto a esas fuerzas se considera que las mujeres y los hombres que trabajan percibirán cada vez más su propia situación laboral desde una perspectiva mundial. Estos cambios, además de afectar a las economías de los países, tienen consecuencias sobre la población laboral activa, pues influyen en las opciones de empleo; las relaciones sociales o familiares; las necesidades de formación; la actualización de conocimientos y destrezas; los ritmos de trabajo; la disponibilidad de recursos; la ordenación de los procesos laborales; los cambios en las ofertas laborales, y también sobre la salud de la población.

Debido a estas variaciones observamos que el perfil de las enfermedades y accidentes laborales ha cambiado

Unidad de Investigación Psicosocial de la Conducta Organizacional (UNIPSICO), Universitat de València. Valencia, España.

Psicólogo doctor en Psicología

Recibido: 08-05-12 Aprobado: 30-05-12 
en las últimas décadas hacia una situación en la que los riesgos de origen psicosocial, y sus consecuencias, han cobrado protagonismo por el incremento de la frecuencia con que aparecen implicados en el origen de las bajas laborales, ocasionadas por problemas de salud con origen en el trabajo, o la accidentabilidad laboral.

\section{FACTORES Y RIESGOS PSICOSOCIALES DERIVADOS DEL TRABAJO}

Los factores psicosociales son condiciones presentes en situaciones laborales relacionadas con la organización del trabajo, el tipo de puesto, la realización de la tarea, e incluso con el entorno; que afectan al desarrollo del trabajo y a la salud de las personas trabajadoras. Los términos "organización del trabajo" y "factores organizativos" son intercambiables en el contexto laboral con "factores psicosociales" para señalar las condiciones de trabajo que conducen al estrés ${ }^{(1)}$.

Los factores psicosociales pueden favorecer o perjudicar la actividad laboral y la calidad de vida laboral de las personas. En el primer caso fomentan el desarrollo personal de los individuos, mientras que cuando son desfavorables perjudican su salud y su bienestar.

En este caso hablamos de riesgo psicosocial, que es fuente de estrés laboral, o estresor, y que tiene el potencial de causar daño psicológico, físico, o social a los individuos ${ }^{(1)}$.

Los riesgos psicosociales con origen en la actividad laboral pueden estar ocasionados por un deterioro o disfunción en:

a) Las características de la tarea: cantidad de trabajo, desarrollo de aptitudes, carencia de complejidad, monotonía o repetitividad, automatización, ritmo de trabajo, precisión, responsabilidad, falta de autonomía, prestigio social de la tarea en la empresa, etc.

b) Las características de la organización: variables estructurales (tamaño y diferenciación de unidades, centralización, formalización), definición de competencias, estructura jerárquica, canales de comunicación e información, relaciones interpersonales, procesos de socialización y desarrollo de la carrera, estilo de liderazgo, tamaño, etc.

c) Las características del empleo: diseño del lugar de trabajo, salario, estabilidad en el empleo y condiciones físicas del trabajo. d) La organización del tiempo de trabajo: duración y tipo de jornada, pausas de trabajo, trabajo en festivos, trabajo a turnos y nocturno, etc.

La percepción de riesgo no se concreta siempre en percepción de riesgo físico, también puede ser riesgo psicológico (por ejemplo: despido, pérdida de estatus, pérdida de prestigio, etc.).

La exposición a este tipo de riesgos no deteriora necesariamente la salud del trabajador, aunque como su nombre indica son una fuente de riesgo, pues si el individuo utiliza unas estrategias de afrontamiento funcionales podrá manejar la situación laboral para eliminar el riesgo, o podrá modificar su comportamiento, sus cogniciones o su emociones para adaptarse a la situación y convivir con ella. Por esto, es importante desarrollar programas de formación en materia de prevención junto a otro tipo de acciones dirigidas a eliminar las fuentes de riesgo. No obstante, y debido a que se ha demostrado que los riesgos psicosociales en el trabajo suelen tener carácter crónico, la exposición a situaciones de riesgo psicosocial supone una tensión psicológica continua para todos los individuos.

El origen del problema en las situaciones de riesgo psicosocial no está en el individuo, sino que suele estar en el entorno que es de donde provienen dichas situaciones de riesgo debido a un mal diseño y ordenación del trabajo. Esto es, los riesgos psicosociales en el trabajo aparecen porque se generan unas condiciones laborales difíciles de tolerar para la mayoría de los trabajadores. Obviamente, podemos encontrar individuos que presentan unas cualidades personales superiores al resto de sus compañeros, pero no todos los seres humanos somos capaces de batir un récord olímpico. Por ello, es necesario evaluar el entorno e intentar modificarlo para generar unas condiciones de trabajo adecuadas para el "trabajador normal" que debe desempeñar el puesto.

Por este motivo, las condiciones laborales de exposición crónica a riesgos psicosociales van más allá de ser un problema individual, y pueden constituir un verdadero problema de salud pública cuando su tasa de prevalencia e incidencia resulta alta. La promoción de la salud laboral es una estrategia de intervención importante para fomentar la salud de los trabajadores y, consecuentemente, de la población.

\section{RIESGOS PSICOSOCIALES EMERGENTES EN EL TRABAJO}

Los cambios en el mundo del trabajo acaecidos en las últimas décadas, han llevado a un incremento en las 
tasas de prevalencia de algunos riesgos psicosociales. El informe publicado por la Agencia Europea para la Seguridad y la Salud en el Trabajo (2) sobre riesgos psicosociales en el trabajo revela que los cambios técnicos $u$ organizativos en el mundo laboral, junto con los cambios socioeconómicos, demográficos y políticos, incluido el fenómeno de la globalización, han dado lugar a la aparición de los denominados riesgos psicosociales emergentes que tienen consecuencias sobre la salud de la población debido a que pueden provocar un mayor estrés laboral, repercutiendo negativamente en la salud y seguridad de los trabajadores.

Un riesgo psicosocial emergente es cualquier riesgo que: a) está causado por nuevos procesos, tecnologías, lugares de trabajo, cambios sociales u organizativos; o b) era un factor conocido, pero se considera como un nuevo riesgo debido a avances científicos o percepciones sociales. Se considera que el riesgo va en aumento cuando: a) el número de situaciones de peligro que producen el riesgo va en aumento; $b$ ) la probabilidad de exposición al riesgo aumenta; o c) los efectos sobre la salud de los trabajadores empeoran ${ }^{(2)}$.

\section{Estos riesgos han sido agrupados en cinco áreas:}

a) Nuevas formas de contratación laboral, caracterizadas por la aparición de contratos de trabajo más precarios junto con la tendencia a la producción ajustada, y la subcontratación e inseguridad en el puesto de trabajo. Los trabajadores con contratos precarios suelen realizar tareas más peligrosas, en peores condiciones, y recibir menos capacitación. La falta de estabilidad laboral y contractual puede aumentar los niveles de estrés y de ansiedad del trabajador.

b) Envejecimiento de la población laboral activa y retraso en la edad de jubilación, que la hace más vulnerable a la carga mental y emocional.

c) Intensificación del trabajo, caracterizado por la necesidad de manejar cantidades de información, y carga de trabajo cada vez mayores y bajo una mayor presión en el ámbito laboral. Este riesgo está presente sobre todo en los campos muy competitivos en los que los trabajadores pueden temer que su eficiencia y su rendimiento se evalúen con mayor detenimiento $y$, por ello, tienden a trabajar más horas para finalizar sus tareas. En ocasiones, puede que no reciban una compensación adecuada por ese aumento de la carga laboral, o que no reciban el apoyo social necesario para poder asumir dicha carga. El estrés laboral provocado por el aumento de la carga laboral, junto con las mayores exigencias sobre un menor número de trabajadores, repercutirían de forma negativa en la salud y la seguridad de los trabajadores.

d) Fuertes exigencias emocionales en el trabajo, junto con un incremento del acoso psicológico y de la violencia, en especial en los sectores de la asistencia sanitaria.

e) Desequilibrio y conflicto entre la vida laboral y personal, debido a la falta de ajuste entre las condiciones de trabajo y la vida privada de las personas. Los empleos precarios, con excesiva carga de trabajo, sumado a horarios laborales variables o impredecibles, sin que se le permita al trabajador ajustarlos a sus necesidades personales; pueden provocar conflictos en la vida profesional y privada del trabajador, con efectos perjudiciales para su salud.

Hay que señalar que siempre han existido riesgos psicosociales en el trabajo; lo que ha cambiado es la percepción social que existe sobre ellos, que genera una diferencia "epidemiológica". Los cambios sociales ocurridos; la nueva ordenación del trabajo; los cambios en los sectores ocupacionales; el cambio de la relación profesional entre los trabajadores y los clientes o usuarios, han contribuido al incremento de este tipo de riesgos, hasta el punto de constituir un problema que, por el número de bajas laborales en las que están implicados, y por las consecuencias que tienen sobre el éxito de una organización merecen ser considerados y tratados por las autoridades que regulan el mundo del trabajo y de la salud.

\section{PREVALENCIA DE LOS RIESGOS PSICOSOCIALES EN EL TRABAJO Y SUS CONSECUENCIAS}

En la VI Encuesta de Condiciones de Trabajo en España elaborada por el Instituto Nacional de Seguridad e Higiene en el Trabajo, los riesgos psicosociales en el trabajo fueron una de las principales causas de enfermedades y de accidentes laborales (3); tal es así que el $70,9 \%$ de los trabajadores encuestados señalaba riesgos para tener accidentes laborales. En algunos colectivos ocupacionales este porcentaje es algo más elevado. En el caso del personal sanitario el porcentaje asciende al $74,9 \%$.

El análisis de las causas de accidentes reveló que las principales causas de los accidentes se debían a distracciones, descuidos, despistes o falta de atención $(45 \%)$, trabajar muy rápido $(19,4 \%)$, y al cansancio o fatiga $(17,8 \%)$. En el estudio se concluye que los 
trabajadores que se perciben expuestos a factores de riesgo psicosocial (como sobrecarga de trabajo, exceso de carga mental, realización de tareas repetitivas y de muy corta duración) presentan porcentajes de respuesta significativamente mayores en sintomatología psicosomática (como problemas de sueño, cansancio, dolores de cabeza, mareos, etc.) que los no expuestos.

Por otra parte, la Encuesta sobre Calidad de Vida Laboral realizada en 2010 por el Ministerio de Trabajo e Inmigración ${ }^{(4)}$ del Gobierno de España, concluye que el $49,9 \%$ de las personas ocupadas manifiestan niveles altos o muy altos de estrés frente al $17,4 \%$ que expresan niveles bajos o muy bajos.

La conclusión a la que han llegado estudios realizados en la Unión Europea (UE) es que los riesgos psicosociales son un problema importante debido al coste económico y social que suponen. Según la Quinta Encuesta Europea sobre Condiciones de Trabajo ${ }^{(5)}$ alrededor del $60 \%$ de los trabajadores encuestados manifestaron problemas relacionados con el exceso de carga de trabajo, y un $19 \%$ percibían falta de apoyo social en el trabajo. Respecto a las acciones violentas, como agresiones verbales, insultos, amenazas y humillaciones, la media de los países de la Unión Europea se situó en torno a un 14\% de trabajadores que manifestaron haberlas sufrido en el último año. Otro riesgo relevante de la encuesta fueron las tasas de exposición a trabajo emocional. En algunos sectores ocupacionales como sanidad, la exposición a este riesgo presentó una alta tasa de prevalencia, pues cerca del $40 \%$ de los trabajadores manifestaron tener problemas relacionados con el trabajo emocional. Como consecuencia de la exposición a estos y otro tipo de riesgos un $20 \%$ de los trabajadores encuestados en los 27 países de la UE informaron que sus condiciones de trabajo suponían un riesgo para su salud mental.

No es de extrañar que, según recoge la Agencia Europea para la Seguridad y Salud en el Trabajo (http:// osha.europa/en/topics/stress), el estrés sea el segundo problema de salud relacionado con el trabajo informado con más frecuencia, afectando en 2005 al $22 \%$ de los trabajadores de la UE. Según estimaciones hechas en el 2002 el problema costaba a la UE, formada entonces por 15 países, un mínimo de $€ 20000$ millones al año en relación al tiempo perdido y gastos sanitarios.

En Estados Unidos, el estrés laboral constituye un problema similar al de la UE. En un informe del National Institute for Occupational Safety and Health (NIOSH), entre el 28 al $40 \%$ de los trabajadores informaron que su trabajo resultaba estresante ${ }^{(6)}$; $y$ que aquellos trabajadores con ansiedad, estrés, o alteraciones neuróticas pierden muchos más días de trabajo ( 25 días de promedio perdidos por trabajador) en el año 2001, que aquellos que no presentaban este tipo de alteraciones (solo seis días como promedio perdidos por trabajador) ${ }^{(7)}$.

Los resultados de los estudios realizados permiten concluir que los riesgos psicosociales en el trabajo y sus consecuencias, incluyendo el estrés laboral, suponen un coste económico y social importante, hasta el punto de que la dimensión de este problema se puede considerar una cuestión de salud pública ${ }^{(1,8)}$.

\section{PROMOCIÓN DE LA SALUD EN EL LUGAR DE TRABAJO}

Cada vez son más los estudios que consideran que el lugar de trabajo puede ser un lugar privilegiado para la prevención de trastornos psicológicos y para promover una mejor salud mental.

La Agencia Europea para la Seguridad y la Salud en el Trabajo ${ }^{(9)}$ define la promoción de la salud en el lugar de trabajo como: El esfuerzo en común de empresarios, trabajadores y la sociedad en su conjunto por mejorar la salud y el bienestar laboral de los trabajadores. Para esa organización, ese esfuerzo se puede articular en cuatro tipos de acciones:

1. La mejora de la organización del trabajo mediante medidas que permitan flexibilizar la jornada laboral flexibilizar los puestos de trabajo, por ejemplo mediante el teletrabajo y el trabajo a domicilio; y el aprendizaje permanente mediante la rotación y la diversificación de los puestos de trabajo.

2. La mejora del entorno de trabajo fomentando el apoyo entre compañeros, ofreciendo a los empleados la posibilidad de participar en el proceso de mejora del entorno de trabajo, y brindando alimentación sana en los lugares de trabajo (por ejemplo, comedores, máquinas expendedoras de alimentos, etc.).

3. Fomentar la participación de los empleados en actividades saludables y actividades deportivas.

4. Fomentar el desarrollo personal ofreciendo cursos sobre competencias sociales, manejo del estrés, y programas para evitar tabaquismo o alcoholismo.

\section{PSICOLOGÍA DE LA SALUD OCUPACIONAL}

La disciplina que aborda el estudio de los riesgos psicosociales en el trabajo, y de la calidad de vida laboral es la Psicología de la Salud Ocupacional (PSO) ${ }^{(10)}$. Su 
objetivo es que las personas puedan producir, atender a los demás, desarrollarse, y ser valoradas en el ejercicio de su actividad laboral, además de tener la posibilidad de utilizar su talento, sus conocimientos, y sus destrezas y habilidades, como vía para alcanzar un alto rendimiento, al tiempo que perciben alta satisfacción laboral y bienestar en el trabajo ${ }^{(11,12) .}$

Los profesionales que abordan el estudio de la salud desde esta aproximación conciben su campo de acción diferente de lo que podría definirse como un acercamiento médico al estudio de la salud ocupacional. La PSO es una nueva especialidad de la psicología con carácter básico y aplicado, que resulta de la integración de contenidos en la Salud Pública con la Medicina Preventiva, la Psicología Clínica y la Psicología de la Salud, aplicados en el contexto de las organizaciones laborales ${ }^{(11)}$

Los ambientes saludables y la salud de los trabajadores son condiciones esenciales para la efectividad de las personas y para el rendimiento de las organizaciones. Por ello, la PSO busca cuidar la salud de las personas y desarrollar ambientes saludables, pues ambos frentes son inseparables.

Fomentar la salud psicosocial en el lugar de trabajo es fomentar la salud pública de la población, pues las personas en edad de trabajar pasan la mayor parte de su tiempo de vigilia en contextos laborales, o desarrollando una actividad laboral en su domicilio. Por este motivo, la promoción de la salud laboral y la prevención de las enfermedades y accidentes laborales relacionados con los riesgos psicosociales deberían ser materia de salud pública para los gobiernos. Como ha señalado la Agencia Europea para la Seguridad y la Salud en el Trabajo ${ }^{(9)}$, la promoción de la salud en el lugar de trabajo no es solo cumplir la normativa en materia de seguridad y salud en el trabajo, también se debe fomentar que las empresas e instituciones contribuyan de manera activa a la mejora de la salud mental, al bienestar y a la calidad de vida laboral de sus trabajadores, teniendo en cuenta sus necesidades, fomentando la participación en la organización, y el diseño saludable de los lugares de trabajo.

\section{Fuentes de financiamiento}

Autofinanciado.

\section{Conflictos de interés}

El autor declara no tener ningún conflicto de interés.

\section{REFERENCIAS BIBLIOGRÁFICAS}

1. Gil-Monte PR. Algunas razones para considerar los riesgos psicosociales en el trabajo y sus consecuencias en la salud pública. Rev Esp Salud Publica. 2009;83(2):169-73.

2. European Agency for Safety and Health at Work. Expert forecast on emerging psychosocial risks related to occupational safety and health. Luxembourg: Office for Official Publications of the European Communities; 2007.

3. Instituto Nacional de Seguridad e Higiene en el Trabajo. VI Encuesta de Condiciones de Trabajo. Madrid: MTAS; 2008.

4. Ministerio de Trabajo e Inmigración. Encuesta de Calidad de Vida en el Trabajo [Internet]. Madrid: Ministerio de Trabajo e Inmigración; 2010 [citado el 1 de mayo de 2012]. Disponible en http://www.empleo.gob.es/estadisticas/ecvt/ Ecvt2010/index.htm

5. European Foundation for the Improvement of Living and Working Conditions. Fifth European Working Conditions Survey. Luxembourg: Eurofound, Publications Office of the European Union; 2012.

6. U.S. Department of Health and Human Services, National Institute for Occupational Safety and Health (NIOSH). Stress...at work. Publication No. 99-101. Cincinnati, OH: NIOSH; 1999.

7. U.S. Department of Health and Human Services, National Institute for Occupational Safety and Health (NIOSH). Worker Health Chartbook, 2004. Cincinnati, OH: $\mathrm{NIOSH} ; 2004$.

8. Melamed S, Shirom A, Toker S, Berliner S, Shapira I. Burnout and risk of cardiovascular disease: evidence, possible causal paths, and promising research directions. Psychol Bull. 2006;132(3):327-53.

9. Agencia Europea para la Seguridad y la Salud en el Trabajo. Promoción de la salud en el trabajo para los trabajadores [Internet]. Bilbao: Agencia Europea para la Seguridad y la Salud en el Trabajo; 2010 [citado el 1 de mayo de 2012]. Disponible en: http://osha.europa.eu/es/ publications/factsheets/94

10. Raymond JS, Wood DW, Patrick WK. Psychology doctoral training in work and health. Am Psychol. 1990;45(10):115961.

11. Quick JC. Occupational health psychology: historical roots and future directions. Health Psychol. 1999;18(1):82-8.

12. Quick JC. Occupational health psychology: the convergence of health and clinical psychology with public health and preventive medicine in an organizational context. Professional Psychology: Research and Practice. 1999;30(2):123-28.

Correspondencia: Pedro R. Gil-Monte

Dirección: Av. Blasco Ibáñez 21; 46010 Valencia, España.

Teléfono: (34) 963864564

Correo electrónico: Pedro.Gil-Monte@uv.es 